\title{
Direct Observation of Magnetic Domain Wall Propagation in NiFe Nanowires
}

\author{
Kai He, ${ }^{*}$ David J. Smith, ${ }^{* *}$ and Martha R. McCartney ** \\ * School of Materials, Arizona State University, Tempe, AZ 85287 \\ ** Department of Physics, Arizona State University, Tempe, AZ 85287
}

The development of novel memory [1] and logic [2] devices based on ferromagnetic nanowires (NWs) requires detailed knowledge of domain-wall (DW) nucleation and propagation. Although DW motion can be probed by indirect measurements of hysteresis loops and magnetoresistance changes, direct nanoscale observation of DW configuration and position is essential, but remains challenging. Electron holography provides a powerful approach to quantify nanoscale magnetic fields by retrieving the corresponding phase shifts caused by the sample [3]. In this present study, we have observed and quantified magnetic-field-driven DW propagation in Permalloy (Py: $\left.\mathrm{Ni}_{81} \mathrm{Fe}_{19}\right)$ NWs using Lorentz microscopy and off-axis electron holography. These Py NWs with a trapezoidal notch as DW trapping site, as shown in Fig. 1(a), were fabricated using electron-beam lithography (EBL) and lift-off process followed by thermal evaporation of Py directly onto a 50-nm-thick silicon nitride membrane. A Philips CM 200 FEG TEM was operated in Lorentz mode, and an in situ magnetic field was used to cause DW motion during observation by tilting the sample with the normal objective lens windings slightly excited [4].

The magnetic contribution to the phase shift across a typical NW was extracted from pairs of electron holograms taken at the remanent state, as shown in Fig. 1(b). The phase change from A to B [Fig. 1(c)] indicates uniformly distributed magnetization along the NW, and the effective magnetic thickness is calculated to be $\sim 15 \mathrm{~nm}$. Lorentz microscopy was used to qualitatively detect DWs within the entire NW, where the DWs are visible with black or white (Fresnel) contrast, as shown in Fig. 2(a). Typical DW configurations, including pinning, depinning, attraction, and annihilation, have been indentified to take place during DW propagation. Detailed magnetization distributions were quantitatively extracted from electron holograms, and then converted into magnetic induction maps, as illustrated in Figs. 2(b)-(e). By controlling the initial chirality or sense of rotation of the magnetic field, the transverse DW (TDW) can be pinned at the notch, and then relaxed to a vortex DW (VDW) at remanence [4]. When the field was applied in the opposite direction, the VDW became dissociated from the notch, forming a configuration consisting of a vortex core and an associated TDW. As the field was further increased, the associated TDW moved away from the notch, while another TDW with opposite chirality was initiated from the nucleation pad. The two DWs with opposite chirality attracted each other and finally annihilated to reach the saturation state of the NW [5].

\section{References}

[1] S.S.P. Parkin, et al., Science 320 (2008) 190.

[2] D.A. Allwood, et al., Science 309 (2005) 1688.

[3] M.R. McCartney and D.J. Smith, Annu. Rev. Mater. Res. 37 (2007) 729.

[4] K. He, et al., Appl. Phys. Lett. 95 (2009) 182507.

[5] This work was supported by U.S. Department of Energy under Grant DE-FG02-04ER46168. We acknowledge use of facilities in the John M. Cowley Center for High Resolution Electron Microscopy and the Center for Solid State Electronics Research at Arizona State University. 
(a)

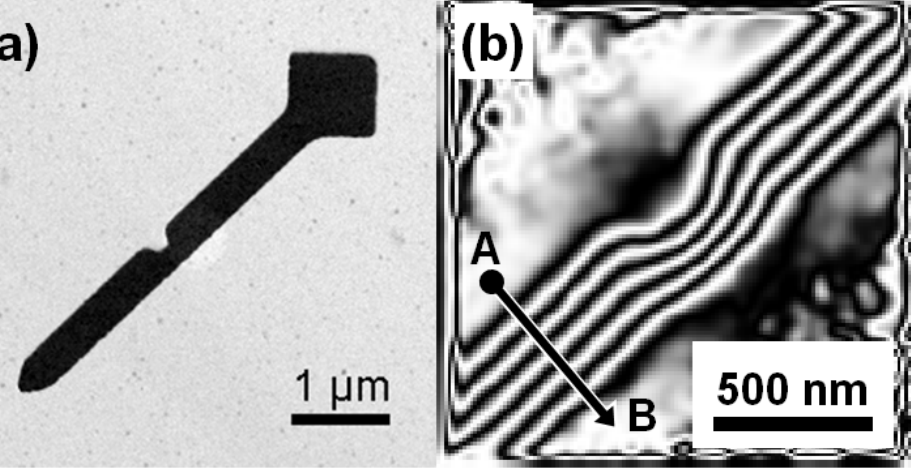

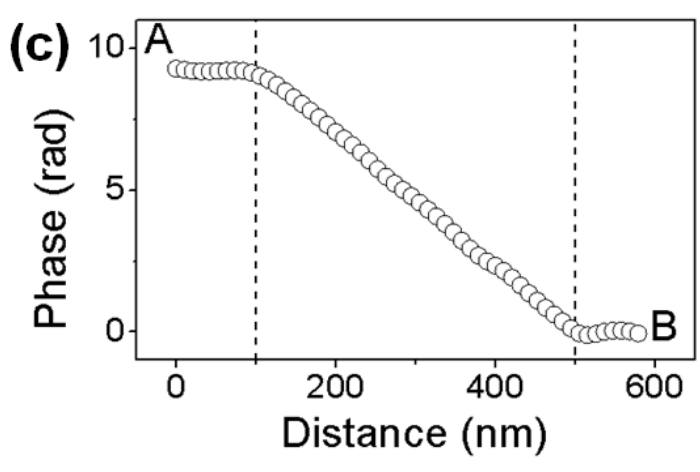

FIG. 1. (a) Lorentz image of Py NW as fabricated; (b) Reconstructed holographic phase image of Py NW around the notch, with contour spacing of $\pi / 2$; (c) Line profile from A to B indicating linear phase shift across the Py NW.
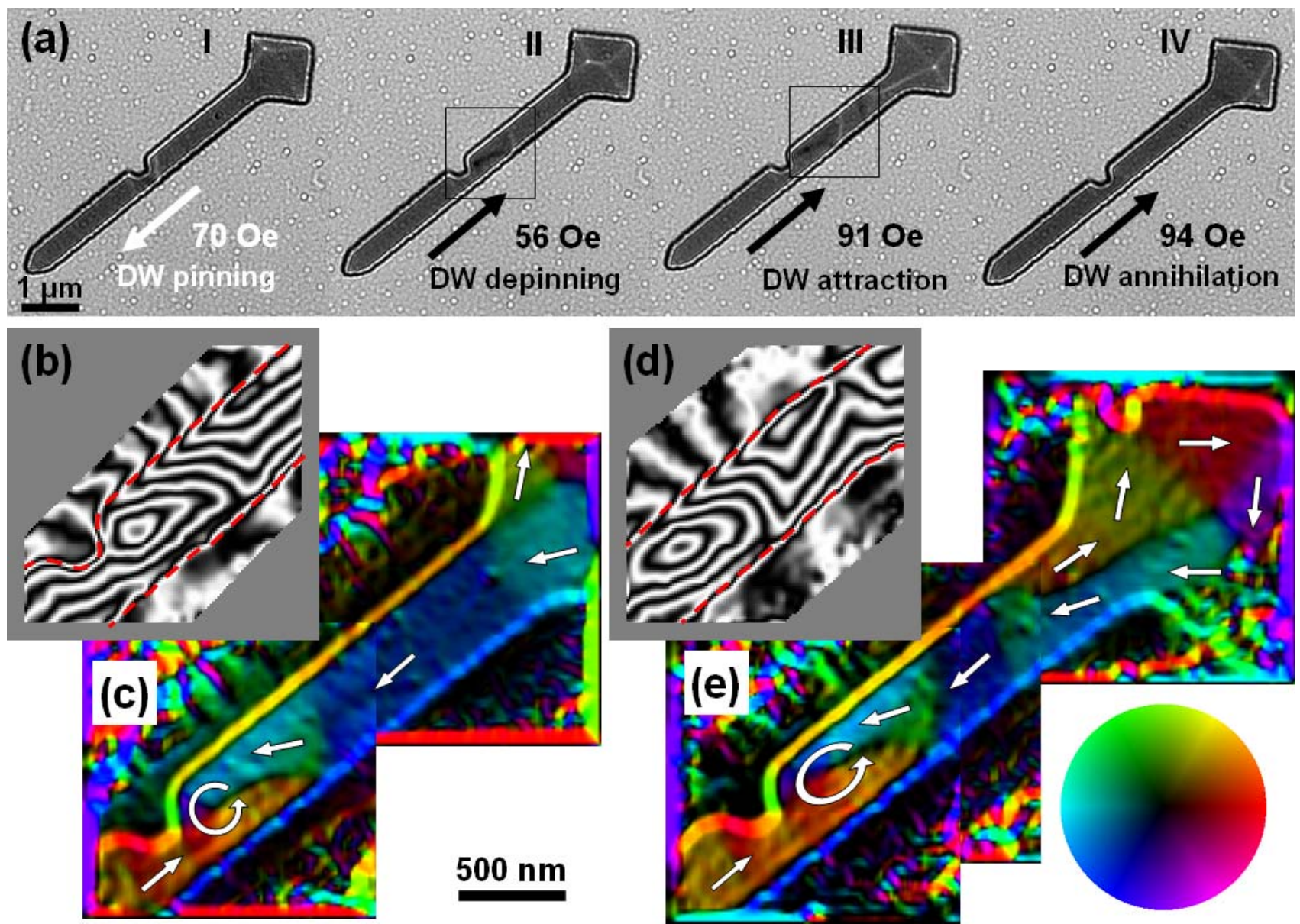

FIG. 2. (a) Defocused Fresnel images showing representative states during DW propagation: (I) DW pinning at the notch, (II) DW depinning from the notch, (III) two DWs attracting each other, (IV) DW annihilation. Applied field direction for each state as indicated by white and black arrows. Holographic phase image and corresponding magnetic induction map of boxed region in state II, and III, shown in (b) (c), and (d) (e), respectively. The NW profile in phase images is indicated by red dash lines. Magnetization directions in magnetic induction maps indicated by color wheel or overlaid arrows. 